\title{
Effects of CS preexposure on inhibition of delay
}

\author{
TODD R. SCHACHTMAN, STEPHEN CHANNELL, and GEOFFREY HALL \\ University of York, York, England
}

\begin{abstract}
Two experiments examined the effects of preexposure to a stimulus on the subsequent acquisition of conditioned suppression by rats. Variations in the level of suppression within conditioning trials were noted so that inhibition of delay (taken here to mean less suppression at the beginning of a trial than at the end) could be detected. Inhibition of delay was observed both during the acquisition of suppression and (in Experiment 1) when suppression began to wane with continued postasymptotic training. Preexposure to the to-be-conditioned stimulus retarded acquisition of suppression and slowed the appearance of inhibition of delay both in acquisition and (in Experiment 1) in postasymptotic performance. Experiment 2 demonstrated that inhibition of delay was attenuated during conditioning that followed preexposure in which the stimulus was paired with a weak reinforcer. These results provide no support for the suggestion that preexposure to a stimulus retards later conditioning because it allows the subject to acquire information about stimulus duration that in turn fosters the development of inhibition of delay. Rather, they are compatible with the suggestion that preexposure causes the stimulus to lose associability.
\end{abstract}

When animals are given extended training in a classical conditioning procedure with a relatively long conditioned stimulus (CS), conditioned responding may come to be restricted to that portion of the CS that is most contiguous with reinforcement (e.g., Pavlov, 1927). A more recent demonstration of the effect was provided by $\mathrm{Zie}-$ linski (1966) in an experiment using the conditioned suppression technique. In each of 21 sessions, rats received three presentations of a 6-min CS followed by electric footshock. Suppression during the CS was virtually complete during the first 10 sessions of training, but thereafter some recovery of responding was observed. This recovery was attributable to the occurrence of responses in the first third of the CS; responding continued to be suppressed toward the end of the CS.

These phenomena have been viewed as instances of inhibition of delay (Pavlov, 1927). This term can be used at a descriptive level and need not imply acceptance of any particular theory of the effect. We use it here to mean less conditioned responding at the beginning of a CS than at the end. It is often assumed, however, that inhibition of delay depends upon subjects' acquiring information about $\mathrm{CS}$ duration and discriminating between the initial and terminal parts of the CS-unconditioned stimulus (US) interval (e.g., Hammond \& Maser, 1970; Millenson \& Dent, 1971).

This analysis has implications for the interpretation of latent inhibition, the retardation of conditioning produced by prior exposure to the to-be-conditioned stimulus ( $\mathrm{Lu}$ bow, 1973). If the duration of the stimulus is held constant during preexposure and conditioning, then informa-

Support for this research was provided by a grant from the UK Science and Engineering Research Council to Geoffrey Hall. We thank Phil Reed for reading a preliminary version of the manuscript, Steve Reilly for assisting with the statistical analyses, and Rob Honey for his comments on this research. Correspondence should be addressed to G. Hall, Department of Psychology, University of York, York YO1 5DD, England. tion about $\mathrm{CS}$ duration is available during the preexposure phase. Possession of such information could facilitate the formation of a temporal discrimination during conditioning between the initial and terminal portions of the CS. Inhibition of delay would then occur rapidly and the overall magnitude of the conditioned response (CR) would be less than that seen in control subjects given no preexposure.

Latent inhibition has been variously attributed to the loss of an attentional response to the stimulus (Lubow, Weiner, \& Schnur, 1981), to a decline in the associability of the stimulus (e.g., Pearce \& Hall, 1980; Wagner, 1978), or to the learning of a zero correlation between the stimulus and other events (Weiss \& Brown, 1974). These explanations have in common the assumption that latent inhibition is an instance of negative transfer from preexposure to conditioning. The hypothesis advanced above challenges this assumption and raises the possibility that latent inhibition may reflect superior learning of a temporal discrimination during the $\mathrm{CS}$, rather than a retardation of conditioning.

The present experiments used a conditioned suppression preparation to assess the effects of CS preexposure on the acquisition of inhibition of delay. Experiment 1 focused on the importance of nonreinforced stimulus preexposure in providing information about $\mathrm{CS}$ duration and thereby influencing the temporal discrimination possibly necessary for inhibition of delay. Experiment 2 examined the contribution of inhibition of delay to the variant of the latent inhibition effect demonstrated by Hall and Pearce (1979) in which the target stimulus is accompanied by a weak reinforcer during the exposure phase.

\section{EXPERIMENT 1}

In the conditioning phase of this experiment, all subjects received training with a relatively long $(120-\mathrm{sec})$ 
stimulus (a tone) preceding electric footshock. The distribution of responding during the CS presentations of the acquisition trials was examined by separately recording responses in each of the four 30-sec components of the CS. In this way, changes in the degree of suppression manifest during a trial might be revealed. Following Zielinski (1966), training was extended to allow assessment of inhibition of delay during the postasymptotic portion of the learning curve when a loss in the CR typically occurs.

There were four groups of subjects that differed in the training they received prior to conditioning. Group Tone-C (for preexposure to a tone of constant duration) received 120-sec nonreinforced presentations of the tone that was to be used as the CS in conditioning. Latent inhibition is to be expected, and thus this group should show retarded conditioning with respect to the control groups, Group VI and Group Light-C. Group VI received equivalent preexposure to the apparatus but with no presentations of the tone. Group Light-C (for light preexposure of constant stimulus duration) received presentations of a light stimulus of the same duration as the target tone preexposure given to Group Tone-C. Preexposure for Group Light-C afforded information about the duration of a nontarget stimulus in the absence of latent inhibition to the target tone. The fourth group, Group Tone-V (for tone preexposure of variable stimulus duration during preexposure), received the same total amount of preexposure to the tone as did Group Tone-C, but the duration of the tone varied from trial to trial during preexposure. Thus, subjects in this group received a latent inhibition treatment in the absence of an opportunity to acquire appreciable information about the duration of the CS used in the conditioning phase. This group was thus less likely than was Group Tone- $\mathrm{C}$ to show enhanced development of inhibition of delay and, inasmuch as inhibition of delay contributes to the latent inhibition effect, was less likely to yield retarded conditioning.

\section{Method}

Subjects. The subjects were 32 naive male adult hooded (PVG) rats, approximately 100 days old. The rats were housed, 2 to a cage, in Perspex cages with the floor layered with wood shavings in a vivarium maintained on a 16-h light/8-h dark cycle. Sessions were run during the light portion of this cycle. The rats were maintained at $80 \%$ of their free-feeding body weights and water was available ad lib in the home cage.

Apparatus. Four identical operant chambers (Campden Instruments, Ltd.) were employed in the study. Each chamber was equipped with a single response lever adjacent to a recessed food magazine to which 45-mg food pellets (Campden Instruments, Ltd.) could be delivered. The floor consisted of steel rods $(0.4 \mathrm{~cm}$ in diameter and spaced $1.4 \mathrm{~cm}$ center to center) that could be electrified by a Campden Instruments (Model 521S) scrambled-current shock source. The roof of the chamber consisted of a sheet of white translucent plastic above which was a 30 -W strip light that could be operated at $100 \mathrm{~V}$ to provide the light stimulus. A 64- $\Omega$ speaker mounted on the rear wall of the box could be used to present either a $4000-\mathrm{Hz}$ tone or a $20 / \mathrm{sec}$ click train at an intensity of $17 \mathrm{~dB}(\mathrm{~A})$ (re: SPL) above a 65-dB(A) background noise level with the inten- sity measured close to the lever. Each chamber was housed in a sound- and light-resistant environmental enclosure containing an exhaust fan that provided background noise.

Procedure. All subjects were initially trained to barpress for food pellets. On Day 1, the subjects were placed in the chambers with the levers removed for $30 \mathrm{~min}$ and noncontingent food pellets were delivered on a variable-time 1 -min schedule with the magazine flaps retracted to allow access to the pellets. Day 2 was identical to Day 1 except that the magazine flaps were in their normal lowered position. On Days 3 and 4, the levers were inserted into the chamber and the subjects were given barpress training on a continuous reinforcement schedule each day until 75 reinforcements had been delivered. On Day 5, the subjects responded on a variable-interval (VI) 30-sec schedule during a 30-min session. On Days 6-10, the subjects barpressed on a VI 60-sec schedule for 30 min each day. Following barpress training, the subjects were assigned to groups balanced for response rates on the final day of the VI 60-sec baseline phase. There were initially 8 subjects per group, but 1 animal in Group Light-C became ill and was eliminated during Phase 1.

Phase 1 was conducted on the baseline and occurred on Days 11-22. During each of these 1230 -min sessions, subjects in Group Tone- $C$ were exposed to two 120 -sec nonreinforced tone presentations, initiated 10 and 22 min into the session. Group Light-C received identical treatment except that the overhead light was substituted for the tone. Group Tone-V received two presentations of the tone in each of these sessions, but the duration of the tone varied within and across sessions in such a way that the average duration over the 12 sessions was $120 \mathrm{sec}$. The first and second tone presentations were 60 and $180 \mathrm{sec}$ long on Days 11 , 15 , and 19; 120 and $90 \mathrm{sec}$ long on Days 12,16, and 20;30 and $210 \mathrm{sec}$ long on Days 13, 17, and 21; and 150 and $120 \mathrm{sec}$ long on Days 14, 18, and 22. The fourth group, Group VI, merely barpressed on the VI 60-sec baseline during this phase. Responses were recorded during each $\mathrm{CS}$ presentation and during a period immediately preceding the $C S$ and equal in duration to the CS presentation for that trial. Additionally, responses were recorded separately during the 30-sec epochs (subtrials) of each CS presentation.

Phase 2 occurred on Days 23-34. In each of these 30-min sessions, all subjects received two 120 -sec presentations of the tone, at 10 and 22 min into the session, with offset of the tones concurrent with the onset of a .4-mA, 500-msec footshock. Phase 2 treatments were superimposed on the VI 60-sec operant baseline and responses were recorded as described for Phase 1.

\section{Results and Discussion}

Daily suppression scores. Daily suppression scores were calculated for each subject, using the ratio $a /(a+b)$ where $a=$ responses emitted during both stimulus presentations and $b=$ responses emitted during both prestimulus periods in that session. After the first session, group mean scores did not deviate substantially from .50 during Phase 1. In the final session of this phase, the group mean scores were .53 for Group Tone-C, .52 for Group Tone-V, and .50 for Group Light-C. These scores did not differ reliably $(F<1)$. They were derived from approximately equivalent baseline response rates. In the final session of Phase 1, the mean rates of response in the absence of the stimulus were 17.19 responses per minute for Group Tone-C, 12.22 for Group Tone-V, 14.82 for Group VI, and 17.14 for Group Light-C $[F(3,27)=1.89, p>.10]$.

Baseline response rates declined during Phase 2, but did so in all groups, and there were no reliable differences among them on this measure. The rates over all 


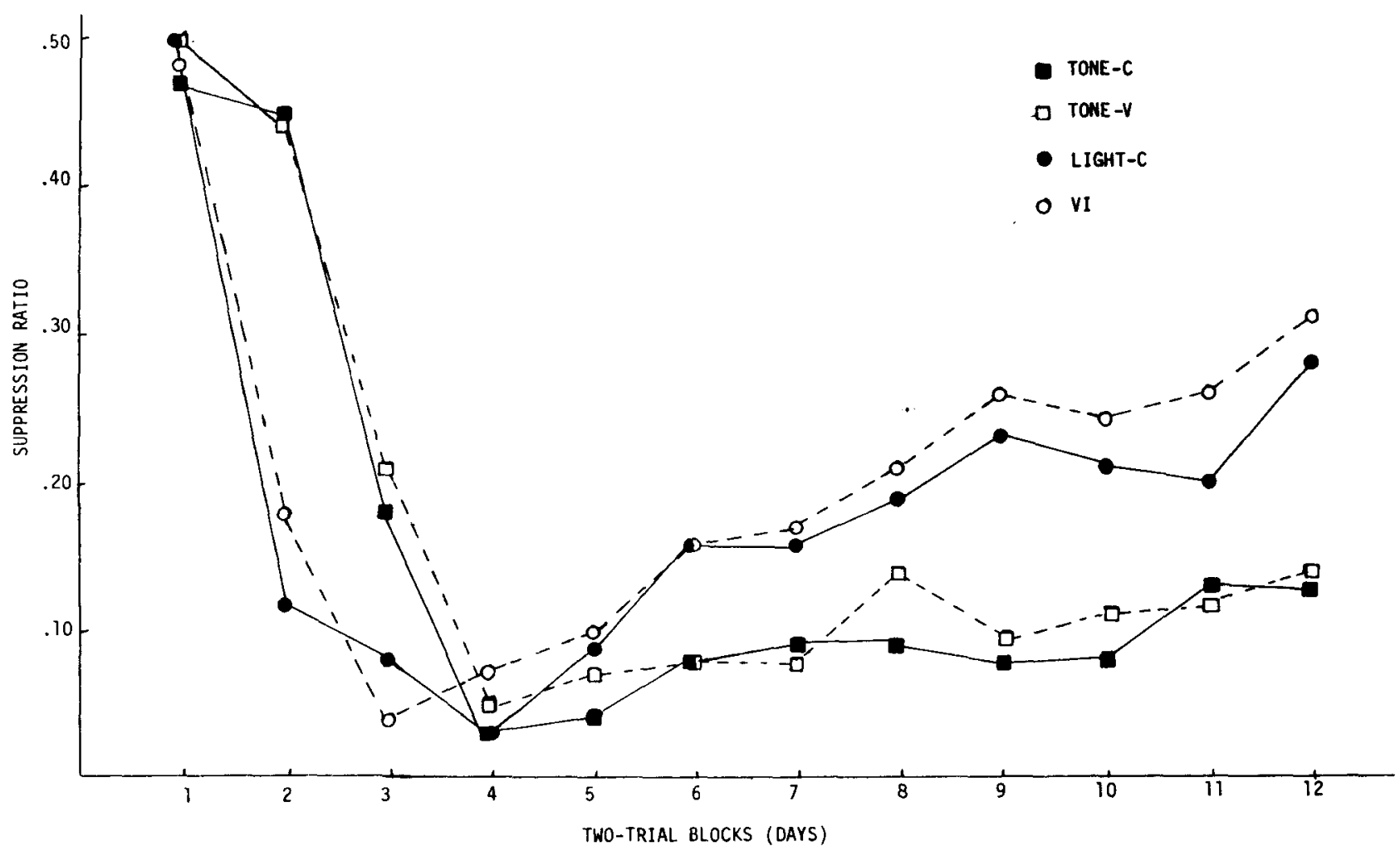

Figure 1. Experiment 1: Group mean daily suppression ratios for Phase 2. Group Tone-C received preexposure to a tone of constant duration, Group Tone-V received preexposure to a tone of variable duration, Group Light-C received preexposure to a light of constant duration, and Group VI received pretraining on a variable-interval schedule only.

Phase 2 sessions in responses per minute were 10.14 for Group Tone-C, 7.25 for Group Tone-V, 11.39 for Group VI, and 10.36 for Group Light-C $[F(3,27)=2.77$, $p>$.05]. Suppression scores for Phase 2 are depicted in Figure 1, which shows that all groups acquired suppression over the first few days of conditioning but that thereafter suppression tended to be lost. The groups fell into two pairs. In the preasymptotic phase (up to Day 4), the two groups given preexposure to the tone acquired suppression less readily than did the other two groups. In the postasymptotic phase (from Day 5 onward), the tone-preexposed groups lost suppression less readily than did the other two groups.

An analysis of variance for the preasymptotic scores of the four groups (i.e., the scores for Days 1-4) revealed a significant difference among the groups $[F(3,27)=$ $5.18, p<.01]$, a significant change over days $[F(3,81)$ $=111.29, p<.01]$, and a significant interaction between these factors $[F(9,81)=4.04, p<.01]$. A separate comparison of Group VI and Group Light-C confirmed that there was no difference between them. It revealed only a significant effect of days $[F(3,39)=80.18, p<.01]$, other $F \mathrm{~s}$ being less than 1 . Thus there is no evidence that preexposure to some different stimulus having the same temporal duration as that to be used as a CS modifies the rate of conditioning to that $\mathrm{CS}$.
Both tone-preexposed groups showed latent inhibition. Comparison of the preasymptotic scores for Group Tone-C and Group VI revealed a significant difference between the groups $[F(1,14)=8.97, p<.01]$, a significant change over days $[F(3,42)=69.47, p<.01]$, and a significant interaction between these factors $[F(3,42)=9.99$, $p<.01]$. A similar comparison between Group Tone-V and Group VI revealed significant effects of groups $[F(1,14)=8.30, p<.05]$ and of days $[F(3,42)=12.54$, $p<.01]$, but no significant interaction $[F(3,42)=1.61]$. The two tone-preexposed groups did not differ from one another. A comparison of their scores over Days 1-4 revealed only a significant effect of days $[F(3,42)=51.95$, $p<.01$; other $F \mathrm{~s}<1]$. These results lend no support, therefore, to the suggestion that the latent inhibition effect depends upon the subjects' having been preexposed to a stimulus of the same duration as the CS.

An analysis of variance conducted on the postasymptotic scores for all four groups (i.e., over the last 8 days of conditioning) showed there to be a significant difference among the groups $[F(3,27)=3.80, p<.05]$ and a significant change over days $[F(7,189)=8.18$, $p<.01]$, but no significant interaction between these factors $(F<1)$. A separate comparison of the scores for Group VI and Group Light-C confirmed that there was no reliable difference between the groups $(F<1)$ and 
no significant interaction between groups and days $(F<1)$. There was, however, a significant change over days $[F(7,91)=6.65, p<.05]$, reflecting the postasymptotic loss of suppression evident in these subjects. A similar analysis comparing the scores for the two tonepreexposed groups showed no significant difference between them $(F<1)$ and no significant interaction between groups and days $(F<1)$. The slight loss of suppression shown by these groups proved to be statistically reliable $[F(7,98)=2.12, p<.05]$. Although the tonepreexposed groups and the control groups both showed some postasymptotic loss of suppression, the extent of the effect was clearly greater in the control groups (Figure 1). This surprising result (given the expectation that a variable that reduces the amount of suppression shown during acquisition might also lead to less profound postasymptotic suppression) merits further investigation. An analysis of variance comparing the pooled results of the tone-preexposed groups with the pooled results of the control groups over the last 8 days of training confirmed the existence of a significant difference between preexposed and control subjects $[F(1,29)=29.42, p<.01]$. There was a significant change over days $[F(7,203)=2.84$, $p<.01]$ and no significant interaction $(F<1)$.

Within-CS effects: Preasymptotic performance. In order to investigate changes in suppression within a CS presentation, suppression ratios were calculated for each of the four 30-sec intervals (subtrials) of each 2-min CS presentation in Phase 2. The prestimulus score was derived from the response rate shown in the 2-min period before each trial. The group mean suppression scores for the subtrials of Trials 2-4 are shown in Figure 2; the scores for Trials 5-7 are shown in Figure 3. Trial 1 was omitted because little suppression occurred on this trial (see Figure 1).

Figures 2 and 3 show that even in these very early conditioning trials, the acquisition of suppression was associated with inhibition of delay, that is, with greater suppression at the end of the CS than at the beginning. A clear effect was present by Trial 3 in Groups VI and Light-C. The tone-preexposed groups acquired overall suppression more slowly, but the effect was evident in these groups by Trial 5 . An analysis of variance conducted on the scores for Trials 2-7 including subtrials as a factor revealed a significant difference among the groups $[F(3,27)=8.44, p<.01]$, significant effects of trial $[F(6,162)=77.49, p<.01]$ and subtrial $[F(3,81)=$ $59.69, p<.011$, and significant interactions of these factors (all $p s<.01$ ).

There is no support, therefore, for the suggestion that the latent inhibition effect seen in the preexposed subjects reflects the rapid development of inhibition of delay. If anything, preexposure tended to retard the development of inhibition of delay. Such a retardation is to be expected solely on the grounds that the control groups acquired overall suppression rapidly and that when suppression first appears it does so toward the end of the CS. In order to detect any effect over and above this, it is necessary to select trials on which the groups to be compared showed equivalent overall levels of suppression. The only suitable trials during the preasymptotic phase were Trial 3, on which Groups Tone-C and Tone-V showed overall suppression ratios of .23 and .20 , and Trial 5 , on which Groups VI and Light-C showed overall suppression ratios

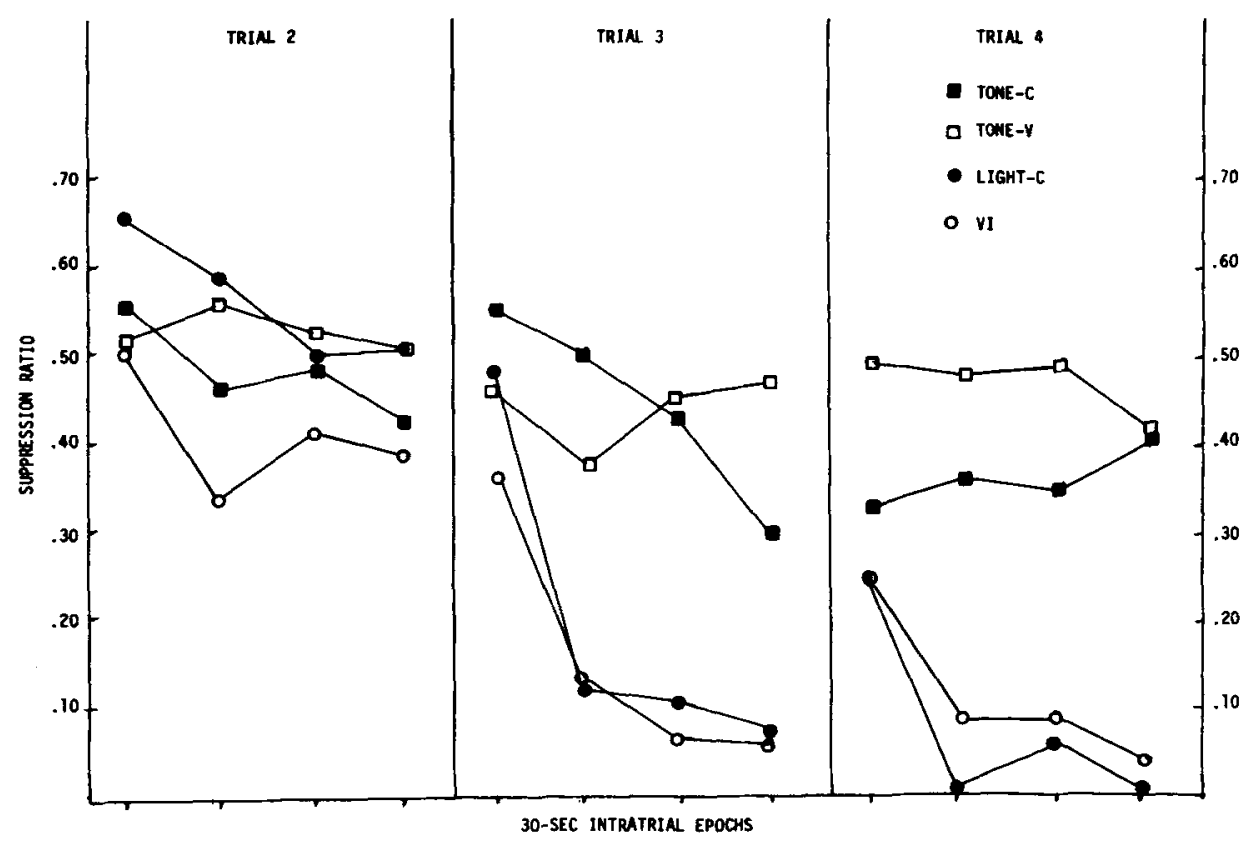

Figure 2. Experiment 1: Group mean suppression ratios for the four 30-sec subtrials of Trials 2-4 in Phase 2. See Figure 1 caption for group descriptions. 
of .18 and .23 . An analysis of variance on the scores for these trials including subtrials as a factor revealed no overall difference among the groups $(F<1)$. There was, however, a significant difference among the subtrials $[F(3,81)=33.05, p<.01]$, and a significant interaction between groups and subtrial $[F(9,81)=3.17, p<.01]$.

A further analysis comparing just the two preexposed groups confirmed the existence of a significant effect of subtrial $[F(3,42)=9.15, p<.01]$ and revealed no significant difference between the groups $(F<1)$ and no significant interaction $[F(3,42)=1.93]$. An equivalent comparison for the two control groups yielded a similar pattern of results-a significant effect of subtrial $[F(3,39)$ $=22.95, p<.01]$, but no other significant effects $(F \mathrm{~s}<1)$. A comparison of the pooled results of the preexposed groups with those of the control groups showed a significant difference in the extent to which they showed inhibition of delay. There was no significant main effect of group $(F<1)$, but there was a significant effect of subtrial $[F(3,87)=32.39, p<.01]$ and a significant interaction between these factors $[F(3,87)=7.32$, $p<.01]$.

Within-CS effects: Postasymptotic performance. The postasymptotic loss of suppression evident in Figure $1 \mathrm{oc}$ curred because of the development of inhibition of delay; that is, when responding during the CS began to reappear after asymptote, it did so especially during the first part of the CS. This effect was more marked in the control groups than in the groups preexposed to the tone, which showed within-CS variations in suppression only toward the very end of training.
Figure 4 shows suppression scores for subtrials over the last three trials of conditioning, when the postasymptotic loss of suppression was maximal. The control groups showed clear inhibition of delay on all three trials; the preexposed groups showed no marked effect until Trial 24.

Statistical analyses were conducted on the subtrial scores pooled over Trials 22-24. An analysis of variance with groups and subtrials as the factors showed a significant difference among the groups $[F(3,27)=3.06$, $p<.05]$, a significant effect of subtrial $[F(3,81)=$ $35.51, p<.01]$, and a significant interaction between the factors $[F(9,81)=2.90, p<.01]$. A separate analysis comparing the two preexposed groups revealed a significant difference among subtrials $[F(3,42)=6.65$, $p<.01]$, but no other significant effects $(F \mathrm{~s}<1)$. A similar comparison of the two control groups confirmed that they did not differ $(F<1)$, although in these subjects too there was a significant effect of subtrial $[F(3,39)$ $=35.27, p<.01]$. The interaction was not significant $[F(3,39)=2.14]$. An analysis comparing the pooled results of the two control groups with those of the two preexposed groups yielded a significant interaction between group and subtrial $[F(3,87)=6.00, p<.01]$, confirming that the control and preexposed groups differed in the extent to which they showed inhibition of delay. There were also significant main effects of group $[F(1,29)$ $=9.35, p<.01]$ and of subtrial $[F(3,87)=35.13$, $p<.01]$.

Summary. Experiment 1 demonstrated that nonreinforced CS preexposures yield a negative transfer effect on the development of inhibition of delay during acquisi-

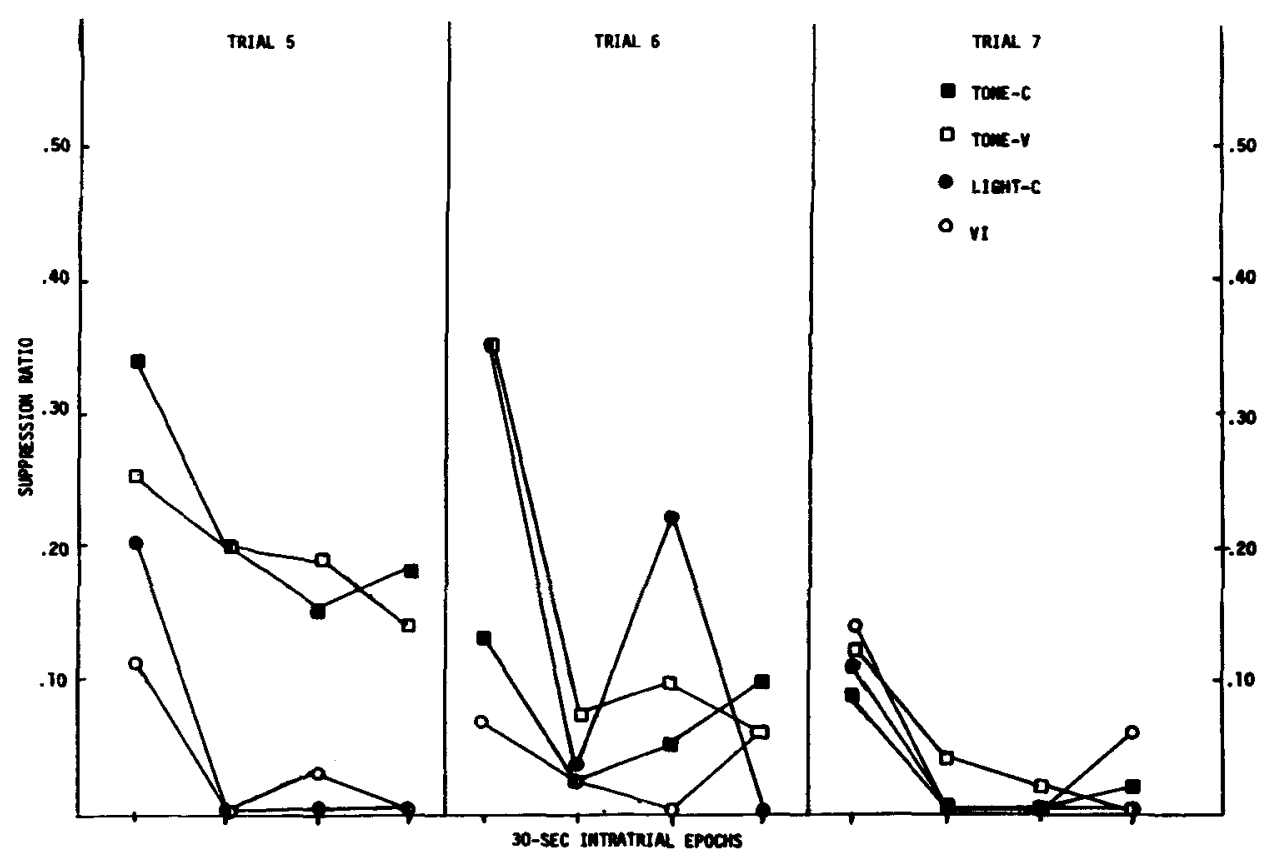

Figure 3. Experiment 1: Group mean supprescion ratios for the four 30-sec subtrials of Trials 5-7 in Phase 2. See Figure 1 caption for group deseriptions. 


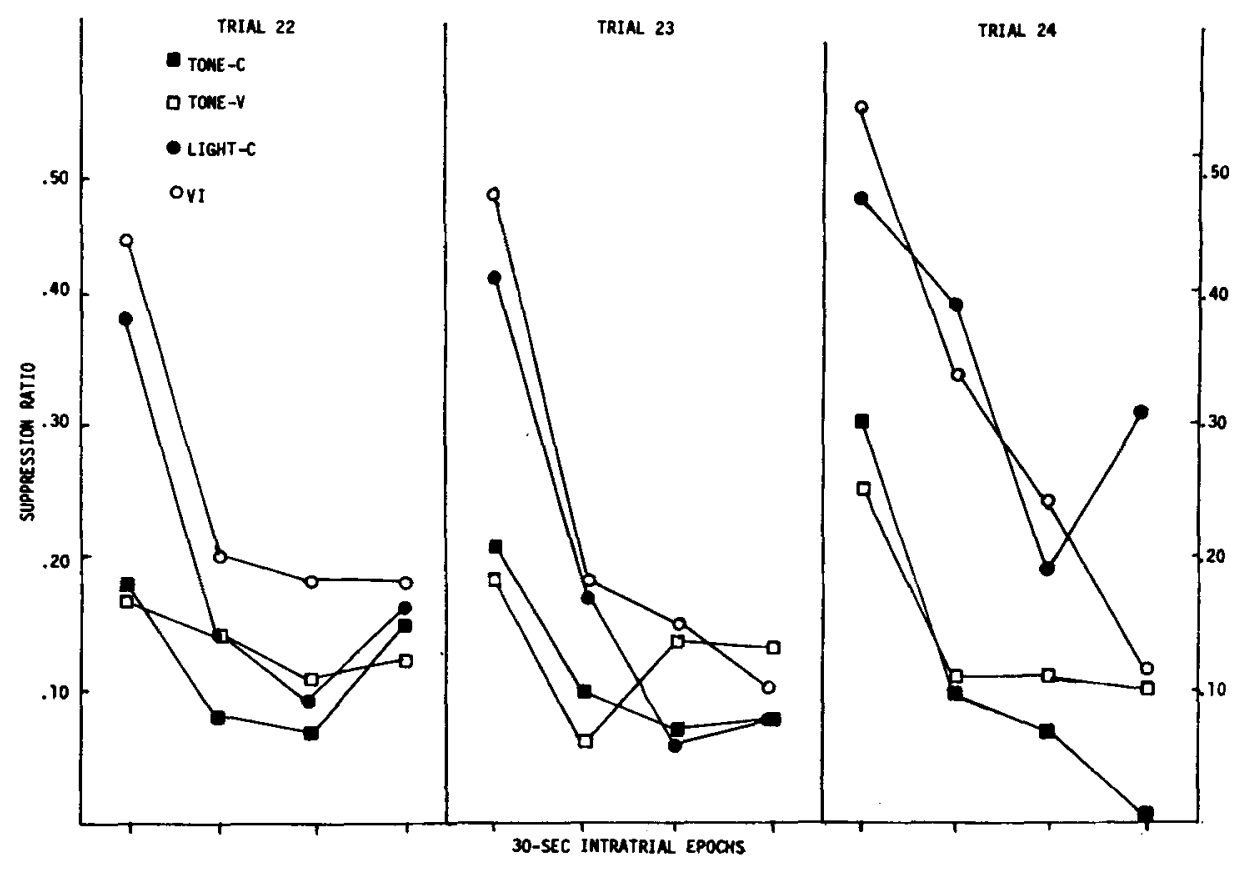

Figure 4. Experiment 1: Group mean suppression ratios for the four 30-sec subtrials of Trials 22-24 in Phase 2. See Figure 1 caption for group descriptions.

tion of conditioned responding, and also during postasymptotic responding. This negative transfer occurred even when the general attenuation of conditioning that occurs with CS preexposure was separated from the inhibition-of-delay effect by comparing groups for the latter using trials in which overall suppression was similar. These results invalidate the suggestion that CS preexposures enable subjects to learn about CS duration, and thereby serve to facilitate the temporal discrimination between the initial and terminal portions of the CS, producing enhanced inhibition of delay. It is necessary to explain, therefore, why CS preexposure should actually attenuate inhibition of delay; this explanation will be given in the General Discussion.

\section{EXPERIMENT 2}

Although the results of Experiment 1 allow us to reject the suggestion that latent inhibition is a consequence of the rapid development of inhibition of delay in preexposed subjects, this notion remains viable as an account of the results of a related preexposure effect reported by Hall and Pearce (1979). In their experiments, Hall and Pearce demonstrated that exposure to a stimulus paired with weak shock produced retarded acquisition of conditioned suppression when the same stimulus was subsequently paired with a stronger shock. Pairings of the CS with weak shock in the first phase of training initially produced some suppression of responding, which was gradually lost as training proceeded (see also Ayres, Moore, \& Vigorito, 1984; Kasprow, Schachtman, \& Miller, 1985). Given the pattern of responding within the conditioning trials in Experiment 1, and the findings of Zielinski (1966), we may conclude that the reinforced preexposure procedure used by Hall and Pearce resulted in the development of considerable inhibition of delay. Subjects given such preexposure might well show transfer of a tendency to develop inhibition of delay, even though subjects given orthodox nonreinforced preexposure do not. Such an effect might be expected on the basis of prior experiments in which an operant analogue of this type of transfer effect was used. The temporal discrimination allegedly required for response scalloping on a fixed-interval (FI) schedule is similar to that required for inhibition of delay (Mackintosh, 1974, p. 171). Trapold, Carlson, and Myers (1965) and Zamble (1969) obtained positive transfer from an initial phase (in which responseindependent food was delivered on a fixed-time [FT] schedule) to the development of response scalloping on an FI schedule in a second phase with the same time base as the FT pretraining. The possibility of positive transfer in classical conditioning challenges Hall and Pearce's assumption that the retarded acquisition produced by their preexposure procedure should be regarded simply as a special case of latent inhibition.

In order to investigate this possibility, the present experiments included the training conditions used by Hall and Pearce (1979) to demonstrate an attenuation of conditioning due to prior pairings of the target CS with weak footshock. One group of subjects received 60-sec presentations of a click train paired with weak shock prior to target conditioning. A second group received nonreinforced click exposures in this phase, and a third group received no CS or US exposures prior to target condi- 
tioning. All subjects were then given pairings of the click with stronger footshock. Responding was recorded during the three 20-sec components of the CS in order to examine changes in suppression during the CS. It was anticipated that the detailed pattern of suppression shown by the groups preexposed to the click alone would match that of the tone-preexposed groups of Experiment 1. But would the group given reinforced preexposure differ and show instead a pattern indicative of enhanced development of inhibition of delay?

\section{Method}

Subjects. The subjects were 24 naive male adult hooded (Lister) rats that were approximately 4 months old. The apparatus was identical to that of Experiment 1 and the subjects were maintained in the same manner as described for that experiment.

Procedure. All subjects were initially trained to barpress for food pellets on Days 1-10 as in Experiment 1, except that baseline barpressing sessions of the VI schedule were $50 \mathrm{~min}$ in duration. Following barpress training, subjects were assigned to groups $(n=8)$ approximately matched in terms of their barpressing rates on the final day of the VI 60-sec baseline phase.

Phase 1 occurred on Days 11-16 of the study and treatments were superimposed on the operant baseline. In each of these 50 -min sessions, subjects in Group Click+ were given six 60-sec presentations of the click train, the offset of which was concurrent with the onset of a .1-mA $500-\mathrm{msec}$ footshock. Trials were spaced $6 \mathrm{~min}$ apart. Group Click- was treated in the same way except that footshocks were omitted. Group VI simply continued to barpress on the VI 60 -sec baseline in the absence of discrete stimulus presentations during this phase.

Phase 2 occurred on Days 17-19. During each of these 50-min sessions, all subjects received three presentations of the 60 -sec click train, with CS offset concurrent with the presentation of .3-mA 500-msec footshock. Responding was recorded for the 60-sec prestimulus and CS periods in Phases 1 and 2. Additionally, responding during the three successive 20-sec epochs that made up the 60sec click train presentations were separately recorded during Phases 1 and 2 .

\section{Results and Discussion}

Phase 1. Daily suppression scores were computed for Groups Click - and Click+, using the total responses emitted by each subject during all CS presentations and all prestimulus periods on each day. The mean score for Group Click - deviated little from .50 across the 6 days of Phase 1 . The performance shown by Group Click+ suggested that a small amount of suppression was initially acquired, but dissipated with further Phase 1 training. The daily scores for the six Phase 1 sessions for this group were $.41, .47, .42, .50, .51$, and .53 . Subtrial suppression scores were calculated for each trial on those days on which Group Click + had a mean suppression score below .50. For this purpose the trial was divided into 20 sec epochs and the prestimulus score used was one third of the total responses recorded in the 60 -sec period preceding presentation of the CS. There was no sign of inhibition of delay. Indeed, on all days, the mean suppression score was somewhat greater for the initial 20-sec epoch (pooled over all six daily trials) than for the final 20-sec epoch. The differences were small, however, and were inconsistent from one subject to another and from one day to another. Presumably reliable within-CS differences in suppression become evident only when suppression is more profound than that seen here.

The three groups were matched for baseline response rate at the beginning of Phase 1 training. The treatments administered in Phase 1 did not influence the groups' baseline rates differentially. Thus, on the last day of this phase, Group Click - had a mean response rate of 14.67 responses per minute; Group Click+, 14.40 responses per minute; and Group VI, 14.12 responses per minute. These scores did not differ significantly $(F<1)$.

Phase 2. Baseline rates of responding were maintained during Phase 2. Over all 3 days of this phase, the rates were $15.98,17.51$, and 14.13 responses per minute for Groups Click-, Click+, and VI, respectively. These scores did not differ significantly $(F<1)$.

Group mean suppression ratios for each trial of Phase 2 are presented in Figure 5. An analysis of variance conducted on these scores revealed a significant difference among the groups $[F(2,21)=10.58, p<.01]$, a significant change over trials $[F(8,168)=32.79, p<.01]$, and a significant interaction between these factors $[F(16,168)$ $=4.22, p<.01]$. It is apparent from the figure that all three groups acquired suppression, but that the groups preexposed to the click did so less readily than Group VI. An analysis comparing Group Click - with Group VI confirmed that a latent inhibition effect had occurred; the analysis yielded a statistically significant main effect of group $[F(1,141)=89.23, p<.01]$ and of trial $[F(8,112)=$ $25.73, p<.01]$, and a significant interaction between these factors $[F(8,112)=8.03, p<.01]$. A comparison of Group Click+ with Group VI revealed a significant effect of group $[F(1,14)=6.48, p<.05]$ and of trial $[F(8,112)=23.98, p<.05]$, and a significant interaction between the two $[F(8,112)=2.47, p<.05]$. This retarded acquisition by Group Click+ replicates previous demonstrations of latent inhibition during reinforced preexposure (Ayres et al., 1984; Kasprow et al., 1985; Hall \& Pearce, 1979). Although retarded with respect to Group VI, Group Click+ acquired suppression more readily than did Group Click-. A comparison of these two groups yielded a significant interaction between trial and group $[F(8,112)=2.62, p<.05]$. There was also a main effect of trial $[F(8,112)=20.74, p<.01]$, but no significant main effect of group $(p>.10)$. This difference between the effects of reinforced and nonreinforced preexposure replicates that reported by Hall and Pearce (1979) and demonstrates that the weak shock of Phase 1 was not without effect.

Subtrial scores were computed for every trial of Phase 2 and are presented in Figure 6. Trial 1 is omitted from the figure because suppression was minimal on this trial. The figure shows that when suppression occurred on a trial, it was almost always more pronounced toward the end of the CS. An additional analysis of variance, including subtrial as a factor, was conducted on the Phase 2 performance of the three groups. This analysis yielded a sig- 


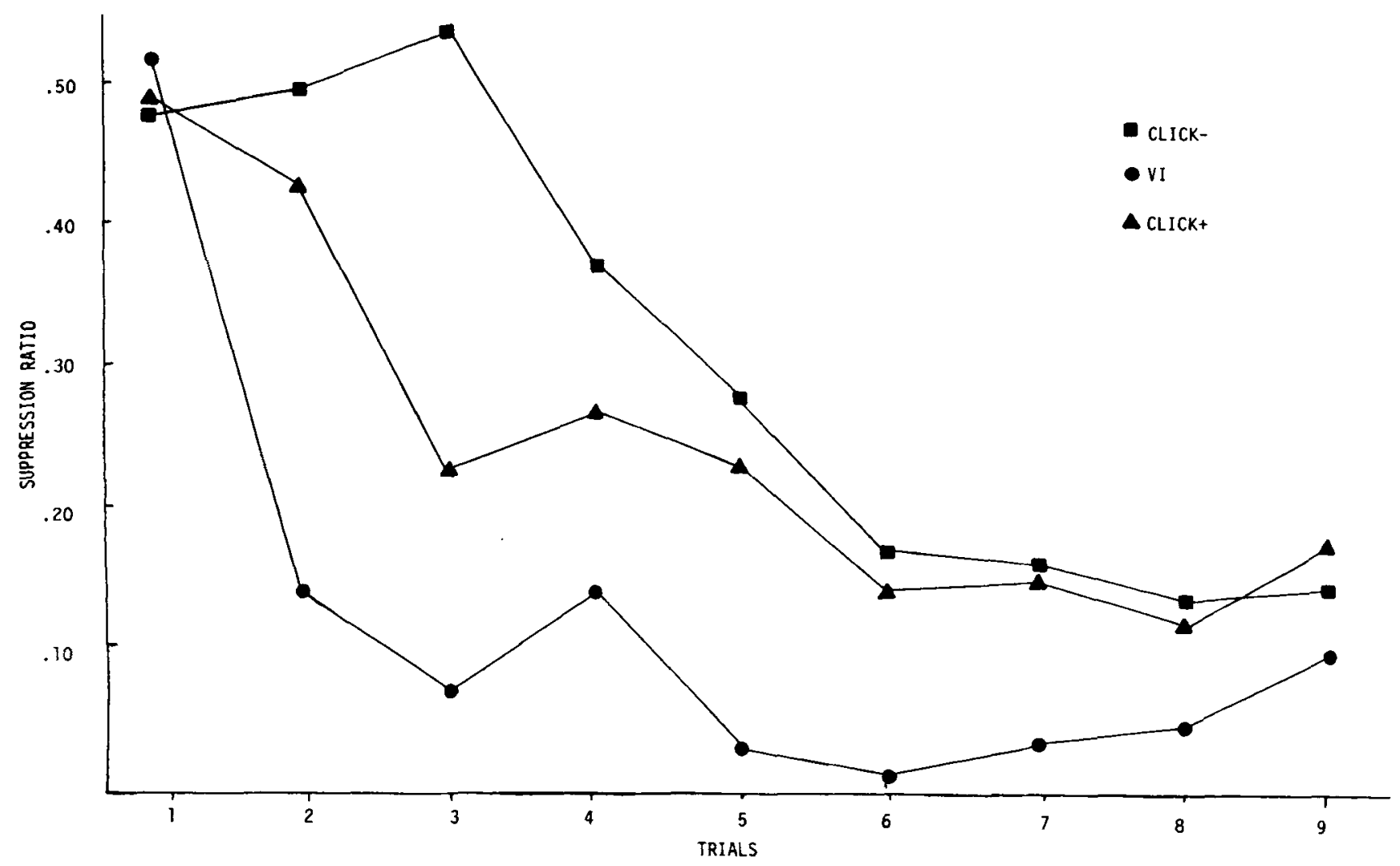

Figure 5. Experiment 2: Group mean suppression ratios for Phase 2. Group Click- received preexposure to a clicker, Group Click+ received preexposure to the clicker preceding a weak shock, and Group VI received pretraining on a variable interval schedule only.

nificant main effect of subtrial $[F(2,42)=20.70$, $p<.01]$, although the interaction between group and subtrial fell short of significance $(p>.10)$, as did the three-way interaction among the factors group, subtrial, and trial $[F(32,336)=1.44, .10>p>.05]$.

Although it falls short of the conventional level of significance, the three-way interaction indicates a tendency for differential suppression across subtrials to appear earlier in training for some groups than for others. Such an outcome is to be expected, given that the groups acquired suppression at differing rates (see Figure 5) and that suppression, when it does appear, appears predominantly in the last portion of the CS.

In order to examine the extent of inhibition of delay in groups of subjects that acquired suppression at different rates, we selected for further analysis trials on which the overall suppression of the various groups was comparable. Since near-complete suppression occurred very rapidly in Group VI, the only suitable trial for this group was Trial 2 (group mean overall suppression score = .14). The preexposed groups achieved this level of suppression by Trial 6, on which the overall scores were .14 for Group Click+ and .19 for Group Click-. An analysis of variance of the subtrial scores for the three groups on these trials revealed no significant main effect of group $(F<1)$, but a significant effect of subtrial $[F(2,42)=$ $6.52, p<.01]$ and a significant interaction between these factors $[F(4,42)=3.68, p<.05]$. Thus, the groups differed in the extent to which they showed within-CS variation in suppression.

A separate comparison of the two preexposed groups on Trial 6 showed that they did not differ from one another $(F<1)$ and revealed no sign of inhibition of delay. There was no significant effect of subtrial $(F<1)$ and no significant interaction between the factors $[F(2,28)=1.98]$. Group Click-, however, differed from Group VI in its pattern of within-CS suppression. A comparison of these two groups revealed no significant main effect of group $[F(1,14)=2.40]$, but a significant effect of subtrial $[F(2,28)=5.75, p<.01]$ and a significant group $\times$ subtrial interaction $[F(2,28)=5.55, p<.01]$. An equivalent comparison between Groups VI and Click+ revealed much the same pattern of results, except that in this case the group $\times$ subtrial interaction fell short of significance $[F(2,28)=2.83, .10>p>.05]$. There was no significant effect of group $(F<1)$, but there was a significant main effect of subtrial $[F(2,28)=7.95, p<.01]$. These findings match those of Experiment 1, with the pre- 

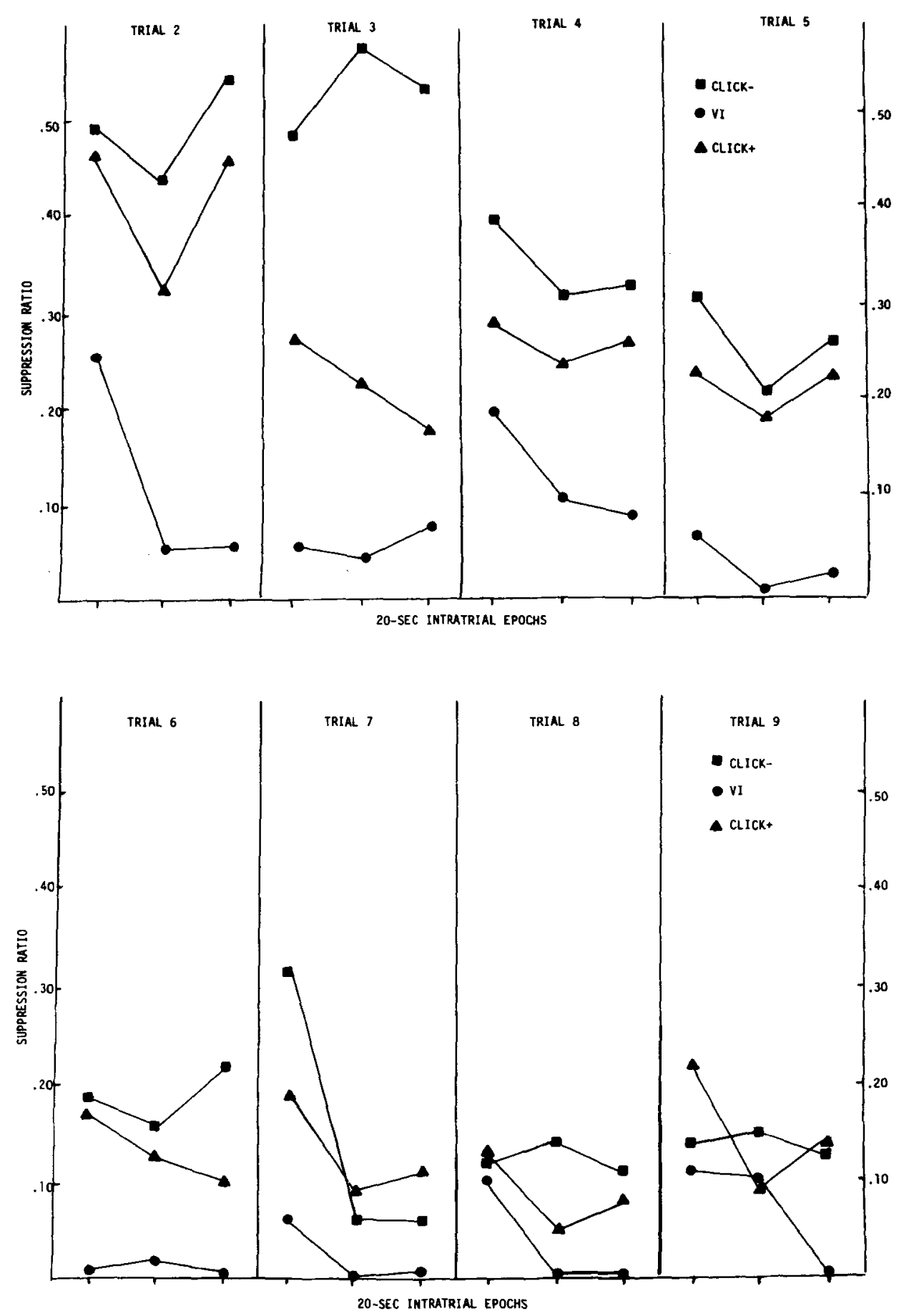

Figure 6. Experiment 2: Group mean suppression ratios for the three 20-sec subtrials of Trials 2-9 in Phase 2. See Figure 5 caption for group descriptions.

exposed subjects showing less of a tendency toward inhibition of delay even when their overall level of suppression had achieved that shown by the control subjects.

In general, the detailed pattern of suppression is much the same for the two preexposed groups and gives no support to the suggestion that reinforced preexposure retards subsequent conditioning by a mechanism different from that underlying the effects of nonreinforced CS preexposure. And in neither case is there evidence that the positive transfer of temporal information acquired during preexposure causes the rapid development of inhibition of delay during Phase 2 conditioning trials. 


\section{GENERAL DISCUSSION}

Animals that have not received preexposure to the tobe-conditioned stimulus show inhibition of delay (i.e., show a smaller CR at the beginning of the CS than at the end) both during the course of acquisition and as the CR begins to wane with prolonged postasymptotic training. The latter finding confirms the results of Zielinski (1966); the former is similar to the results of a number of studies of conditioning of the rabbit's nictitating membrane response (e.g., Coleman \& Gormezano, 1971; Schneiderman \& Gormezano, 1964; Smith, 1968; Smith, Coleman, \& Gormezano, 1969). These studies demonstrated a tendency for the latency of the CR to decrease systematically during the course of acquisition, the onset of the CR being close to the reinforcer on early trials.

One interpretation of postasymptotic inhibition of delay supposes that the subject comes, with extended training, to discriminate the beginning of the CS from the end, and that those cues that define the beginning come to acquire inhibitory properties. It is difficult to imagine such a mechanism's operating during the early trials of acquisition, and the effect seen there is more readily interpreted in terms of the explanation offered for the results of the nictitating membrane experiments. These have been viewed (Gormezano, 1972) as supporting Hull's (1943, 1952) notion that conditioning occurs to the CS trace present at the time of US onset. Conditioned responding then generalizes to other CS trace strengths, causing progressively decreasing latencies of CR onset over trials until CRs may eventually occur throughout the CS.

The hypothesis guiding the experiments reported here was that preexposure to the CS, by allowing the subject to acquire information about its duration, might facilitate the development of "true" inhibition of delay during initial acquisition. It was found, however, that preexposure retarded the development of inhibition of delay, both during the postasymptotic phase and during acquisition. During the postasymptotic phase of Experiment 1, preexposed subjects tended to remain suppressed throughout the whole CS when control subjects at the equivalent stage of training were beginning to show inhibition of delay. During acquisition, preexposed subjects showed no suppression (and hence no inhibition of delay) during early trials, and even when suppression did begin to emerge, the tendency toward an inhibition-of-delay effect was not pronounced.

These results allow the rejection of the suggestion that the latent inhibition effect is a result of enhanced inhibition of delay. It becomes necessary, therefore, to try to explain the present results in terms of our current understanding of the effects of stimulus preexposure. Current theories (e.g., Lubow et al., 1981; Pearce \& Hall, 1980; Wagner, 1978) suppose that preexposure causes a stimulus to lose associability. Since low associability will retard the acquisition of inhibitory as well as excitatory associations (Reiss \& Wagner, 1972; Rescorla, 1971), an explanation for the effects of preexposure on postasymp- totic inhibition of delay can be offered. The lowered associability of the CS will reduce the rate at which inhibitory properties are acquired by the cues that define the beginning of the CS. This explanation assumes, of course, that postasymptotic inhibition of delay reflects true conditioned inhibitory learning (see Rescorla, 1967).

If preasymptotic inhibition of delay reflects a failure of the CR to generalize from the end of the CS to the beginning, then a different account of the effect of stimulus preexposure is required for the preasymptotic effect. Preexposed subjects' slow acquisition of a CR to the cues defining the end of the CS follows from the assumption that preexposure reduces associability. What requires explanation is that the associative strength acquired by the end of the CS should generalize relatively well to the beginning of the CS in preexposed subjects, but relatively poorly in control subjects. If, however, the CS is to be interpreted as consisting of two discriminable halves (beginning and end), then such an outcome might be anticipated by analogy with sensory preconditioning. Preexposure to the CS involves repeated presentation of its two parts, and should allow the formation of an association between them. During subsequent conditioning, associative strength may be acquired only by the later half of the CS, and that rather slowly because of reduced associability of the stimulus. But the existence of the associative link between the cues defining the beginning and the end of the CS should endow the beginning of the CS with the ability to evoke the CR. This account may be speculative, but the fundamental processes involved do not differ in principle from those required to explain other well-documented demonstrations of within-compound learning (e.g., Rescorla, 1980; Rescorla \& Durlach, 1981).

\section{REFERENCES}

Ayres, J. J. B., MoOre, J. W. \& Vigorito, M. (1984). Hall and Pearce negative transfer: Assessments in conditioned suppression and nictitating membrane conditioning experiments. Animal Learning \& Behavior, 12, 428-438.

Coleman, S. R., \& Gormezano, I. (1971). Classical conditioning of the rabbit's (Oryctolagus cuniculus) nictitating membrane response under symmetrical CS-US interval shifts. Journal of Comparative \& Physiological Psychology, 77, 447-455.

Gormezano, I. (1972). Investigations of defense and reward conditioning in the rabbit. In A. H. Black \& W. F. Prokasy (Eds.), Classical conditioning II: Current research and theory (pp. 151-181). New York: Appleton-Century-Crofts.

Ha LL, G., \&EARCE, J. M. (1979). Latent inhibition of a CS during CS-US pairings. Journal of Experimental Psychology: Animal Behavior Processes, 5, 31-42.

HAMMOND, L. J., \& MASER, J. (1970). Forgetting and conditioned suppression: Role of a temporal discrimination. Joumal of the Experimental Analysis of Behavior, 13, 333-338.

Hull, C. L. (1943). Principles of behavior. New York: AppletonCentury-Crofts.

Hull, C. L. (1952). A behavior system. New Haven: Yale University Press.

Kasprow, W. J., Schachtman, T. R., \& Mller, R. R. (1985). Associability of a previously conditioned stimulus as a function of qualitative 
changes in the US. Quarterly Journal of Experimental Psychology, 37B, 33-48.

LuBow, R. E. (1973). Latent inhibition. Psychological Bulletin, 79, 398-407.

Lubow, R. E., Weiner, I., \& SchnuR, R. (1981). Conditioned attention theory. In G. H. Bower (Ed.), The psychology of learning and motivation (Vol. 15, pp. 1-49). New York: Academic Press.

MAckintosh, N. J. (1974). The psychology of animal learning. London and New York: Academic Press.

Millenson, J. R., \& DeNT, J. G. (1971). Habituation of conditioned suppression. Quarterly Joumal of Experimental Psychology, 23, 126-134.

Pavlov, I. P. (1927). Conditioned reflexes. Oxford, England: Oxford University Press.

Pearce, J. M., \& Hall, G. (1980). A model for Pavlovian learning: Variations in the effectiveness of conditioned but not of unconditioned stimuli. Psychological Review, 87, 532-552.

ReISS, S., \& WAGNER, A. R. (1972). CS habituation produces a "latent inhibition effect" but no active "conditioned inhibition." Learning \& Motivation, 3, 237-245.

Rescorla, R. A. (1967). Inhibition of delay in Pavlovian fear conditioning. Journal of Comparative \& Physiological Psychology, 64, 114-120.

RESCORLA, R. A. (1971). Summation and retardation tests of latent inhibition. Journal of Comparative \& Physiological Psychology, 75, 77-81.

Rescorla, R. A. (1980). Pavlovian second-order conditioning: Studies in associative learning. Hillsdale, NJ: Erlbaum.

Rescorla, R. A., \& DURLACH, P. J. (1981). Within-event learning in Pavlovian conditioning. In N. E. Spear \& R. R. Miller (Eds.), In- formation processing in animals: Memory mechanisms (pp. 81-111). Hillsdale, NJ: Erlbaum.

Schneiderman, N., \&ormezano, I. (1964). Conditioning of the nictitating membrane of the rabbit as a function of CS-US interval. Joumal of Comparative \& Physiological Psychology, 57, 188-195.

SMrTh, M. C. (1968). CS-US interval and US intensity in classical conditioning of the rabbit's nictitating membrane. Journal of Comparative \& Physiological Psychology, 66, 679-687.

Smith, M. C., Coleman, S. R., \& Gormezano, I. (1969). Classical conditioning of the rabbit's nictitating membrane response at backward, simultaneous, and forward CS-US intervals. Journal of Comparative \& Physiological Psychology, 69, 226-231.

Trapold, M. A., Carlson, J. G., Myers, W. A. (1965). The effect of noncontingent fixed- and variable-interval reinforcement upon subsequent acquisition of the fixed-interval scallop. Psychonomic Science, 2, 261-262.

W AGNER, A. R. (1978). Expectancies and priming of STM. In S. H. Hulse, H. Fowler, \& W. K. Honig (Eds.), Cognitive processes in animal behavior. Hillsdale, NJ: Erlbaum.

Weiss, K. R., \& Brown, B. L. (1974). Latent inhibition: A review and a new hypothesis. Acta Neurobiologiae Experimentalis, 34, 491-504.

ZAMBLE, E. (1969). Conditioned motivational patterns in instrumental responding of rats. Journal of Comparative \& Physiological Psychology, 69, 536-543.

ZIELINSKI, K. (1966). "Inhibition of delay" as a mechanism of the gradual weakening of the conditioned emotional response. Acta Biologiae Experimentalis, 26, 407-418.

(Manuscript received October 7, 1986; revision accepted for publication May 1, 1987.) 